\section{THU0192 COMBINATION THERAPY OF LEFLUNOMIDE (LEF) AND METHOTREXATE (MTX) IS EFFECTIVE AND WELL TOLERATED IN RHEUMATOID ARTHRITIS (RA) PATIENTS INADEQUATELY RESPONDING TO METHOTREXATE (MTX) ALONE}

${ }^{1} \mathrm{JM}$ Kremer, ${ }^{2} \mathrm{M}$ Genovese, ${ }^{3} \mathrm{GW}$ Cannon, ${ }^{4} \mathrm{R}$ Caldwell, ${ }^{5} \mathrm{~J}$ Cush, ${ }^{6} \mathrm{ME}$ Luggen, ${ }^{7} \mathrm{DE}$ Furst, ${ }^{8} \mathrm{~B}$ Crawford, ${ }^{9} \mathrm{~J}$ Bathon. ${ }^{1}$ Rheumatology, Albany Medical College, Albany, USA; ${ }^{2}$ School of Medicine, Stanford University, Palo Alto, USA; ${ }^{3}$ VA Medical Center, University of Utah, Salt Lake City, USA; ${ }^{4}$ Florida Arthritis \& Allergy Institute, Daytona Beach, USA; ${ }^{5}$ Presbyterian Hospital, Dallas, USA; ${ }^{6}$ University of Cincinnati Medical Center, Cincinnati, USA; ${ }^{7}$ Virginia Mason Research Center, Seattle, USA; ${ }^{8}$ MAPI Values, Boston, USA; ${ }^{9}$ The Johns Hopkins Medical Institutions, Baltimore, USA

\subsection{6/annrheumdis-2001.1094}

Background Combination therapy for the treatment of patients with RA may improve response rate in those who have responded inadequately to MTX alone. As LEF is increasingly being used in RA management, it is relevant to determine the impact of adding LEF to MTX.

Objectives To investigate the benefits of adding LEF to an existing MTX regimen on efficacy, tolerability, physical function, and health-related quality of life (HRQOL) in patients with active RA in a 24-week multi-centre, randomised, double-blind placebo (PLC)-controlled trial.

Methods Subjects with active RA receiving MTX (15?20 mg/ week) for $>=6$ months and at a stable dose for $>=8$ weeks were randomised to receive LEF $100 \mathrm{mg}$ QD $\times 2$ days followed by $10 \mathrm{mg}$ QD thereafter $(\mathrm{n}=130)$ or matching PLC $(\mathrm{n}=133)$. Mean doses of MTX were $16.7 \mathrm{mg} /$ week and $16.2 \mathrm{mg} /$ week for LEF + MTX and PLC +MTX, respectively. The primary efficacy endpoint was ACR 20 responder rate at 24 weeks, with a secondary endpoint of ACR 20 response using LOCF, with both analysed in the ITT population. ACR 20, 50 and 70 response rates, as well as safety monitoring, were assessed every

2 weeks up to week 8 , and every 4 weeks thereafter. The Health Assessment Questionnaire (HAQ) and Medical Outcomes Survey Short Form (SF-36) questionnaire were administered and the mean improvements from baseline were compared at week 24 for the LEF+MTX group and the PLC+MTX group.

Results Using the LOCF method, the ACR 20, 50 and 70 rates were LEF + MTX $=51.5 \%, 26.2 \%$ and $10.0 \%$ vs PLC + MTX $=$ 23.3\%, 6.0\% and 2.3\% (p $<=0.0001, \mathrm{p}<=0.0001$, and $\mathrm{p}$ $<=0.02$ ). At week 24, ACR 20 responder rates were LEF $+\mathrm{MTX}=46.2 \%$ vs PLC+MTX $=19.5 \%$ ( $<<=0.0001)$. Mean baseline values for the disability index (DI) derived from the HAQ were $\mathrm{LEF}+\mathrm{MTX}=1.6$ vs PLC $+\mathrm{MTX}=1.5$ (NS). At 24 weeks, mean changes from baseline in HAQ DI were LEF + MTX $=-0.42$ vs PLC + MTX $=-0.09$ ( $<<=0.0001)$; representing mean improvements of $29 \%$ and $5 \%$, respectively, based on individual percent changes. Mean baseline values for the physical component summary scale (PCS) derived from the SF36 were $\mathrm{LEF}+\mathrm{MTX}=28.4$ vs $\mathrm{PLC}+\mathrm{MTX}=29$ (NS). At 24 weeks, mean changes from baseline in the PC were LEF+MTX $=6.8$ vs PLC + MTX $=0.3(\mathrm{p}<=0.0001)$; representing mean improvements of $29 \%$ and $3 \%$, respectively, based on individual percent changes. Reported AEs included diarrhoea (LEF +MTX $=25.4 \%$ vs PLC + MTX $=13.5 \%$ ), upper respiratory infections $(\mathrm{LEF}+\mathrm{MTX}=22.3 \%$ vs PLC $+\mathrm{MTX}=24.1 \%)$, nausea $(\mathrm{LEF}$ $+\mathrm{MTX}=16.2 \%$ vs PLC + MTX $=11.3 \%)$ and dizziness (LEF $=$ MTX $=7.7 \%$ vs PLC + MTX $=5.3 \%)$. Increases in LFTs of $>3 \times$ ULN at any time during the 24 -week treatment period for ALT and AST for LEF+MTX were 3.8\% and 1.5\%, respectively, compared to $0.8 \%$ on both for PLC+MTX.
Conclusion LEF combined with an existing MTX regimen in patients with active RA is well tolerated and confers significant therapeutic advantages in terms of both efficacy and quality of life.

\section{THU0193 DETERMINATION OF THE POTENTIAL FOR AN IN VITRO PROTEIN BINDING INTERACTION BETWEEN THE LEFLUNOMIDE (LEF) METABOLITE, A77 1726 AND THE SULFASALAZINE (SSZ) METABOLITE, SULFAPYRIDINE (SP)}

DL Larsen, J Holsapple. Biopharmaceutical Sciences, Quintiles, Kansas City, USA

\subsection{6/annrheumdis-2001.1095}

Background LEF is a relatively new DMARD licensed for the treatment of rheumatoid arthritis (RA). When orally administered, it is rapidly and almost completely converted into its active metabolite, A77 1726, which is highly bound to plasma proteins (>99\%). A77 1726 may therefore be subject to interactions with other highly bound drugs. SSZ is also a DMARD used in the treatment of RA. It is metabolised to 5-aminosalicylate (5-ASA) and SP with the latter thought to be responsible for the toxicity of SSZ. SP may also bind to plasma protein, thus there is a possibility of a protein binding interaction. Competitive displacement of bound drug molecules from the binding site may alter the pharmacokinetics of A77 1726 or SP and possibly alter the tolerance of a combined regimen.

Objectives This study investigated the potential for a protein binding interaction between the LEF metabolite, A77 1726 and the SSZ metabolite, SP.

Methods The free and bound fractions of 14C-labelled A77 1726 and 14C-labelled SP were determined by dialysis of drug fortified human plasma against phosphate buffer $(\mathrm{pH} 7.4)$ at 37 degrees celcius. The extent of equilibration was determined after $4,6,8,10$ and $12 \mathrm{~h}$ of dialysis and the time to equilibration was evaluated at concentrations of 10 and $50 \mu \mathrm{g} / \mathrm{ml}$ for both A77 1726 and SP. The extent of binding of 14C-labelled A77 1726, alone, and in the presence of 10,20 and $50 \mu \mathrm{g} / \mathrm{ml}$ of nonlabelled SP was determined at nominal A77 1726 concentrations of 10,20 and $50 \mu \mathrm{g} / \mathrm{ml}$. Conversely the extent of $14 \mathrm{C}$-labelled SP binding alone, or in the presence of 10,20 and $50 \mu \mathrm{g} / \mathrm{ml}$ of non-labelled A77 1726 was determined at nominal SP concentrations of 10,20 and $50 \mu \mathrm{g} / \mathrm{ml}$.

Results No time-dependent changes in A77 1726 and SP free fractions were observed over the 4 to 12 -hour sampling period so a dialysis time of six hours was chosen for the subsequent interaction experiments. Under study conditions, 14C-labelled A77 1726 was $99.2 \%$ to $99.3 \%$ protein-bound over the concentration range 10 to $50 \mu \mathrm{g} / \mathrm{ml}$. The free fractions of A77 1726 tended to be slightly greater at $50 \mu \mathrm{g} / \mathrm{ml}(0.78 \%)$ than at 10 or $20 \mu \mathrm{g} / \mathrm{ml}(0.73 \%$ and $0.74 \%$, respectively). Addition of SP to the plasma had no effect on the observed binding of A77 1726. In contrast to A77 1726, 14C-labelled SP was only 24 to $28 \%$ bound over a concentration range of 10 to $50 \mu \mathrm{g} / \mathrm{ml}$. Free fractions of SP were relatively constant $(73 \%$ to $76 \%)$ as the concentration was increased from 10 to $50 \mu \mathrm{g} / \mathrm{ml}$. Addition of A77 1726 to plasma to provide concentrations of 10,20 and $50 \mu \mathrm{g} /$ $\mathrm{ml}$ had no effect on the observed binding of SP. In addition, the free fractions of SP did not change $(72$ ? $76 \%)$ in the presence of 0, 10, 20 and $50 \mu \mathrm{g} / \mathrm{ml}$ A77 1726.

Conclusion Free fractions of both A77 1726 and SP did not change when each underwent dialysis in the presence of the 
other at concentrations of 10,20 and $50 \mu \mathrm{g} / \mathrm{ml}$. The low binding of SP in human plasma suggests there is a low potential for a protein binding interaction between A77 1726 and SP over the range of concentrations examined. A clinical study to investigate the pharmacokinetics of A77 1726 and SP in healthy subjects is underway.

\section{THU0194 DIGITAL VASCULITIS IN A PATIENT WITH RHEUMATOID ARTHRITS: GOOD RESPONSE ON ANTI-TNF BLOCKADE}

FH Van den Hoogen, MM Zandbelt, A Den Broeder, LB Van de Putte. Rheumatology, University Medical Center, Nijmegen, Netherlands

\subsection{6/annrheumdis-2001.1096}

Background Rheumatoid arthritis (RA) may be complicated by vasculitis. Vasculitis usually affects small vessels of the skin causing nailfold infarcts, but may also affect larger vessels and cause severe damage to internal organs. In such cases, treatment with high doses of corticosteroids or other immunosuppressive drugs may be necessary. TNF-alpha blockade has been shown to be an effective and safe treatment for RA, but thus far no reports have addressed the effect of TNF-alpha blockade on extra-articular manifestations of RA, such as vasculitis.

Objectives We report a patient with RA and nailfold infarcts which repeatedly disappeared for several weeks following monthly i.v. injections with an anti-TNF alpha receptor fusion protein.

Methods A 46 year old woman was diagnosed as having rheumatoid factor positive, erosive RA in 1982. Due to the uncontrollable disease she was included in 1994 in a study with Ro 45 2081, a fusion protein combining two p55 TNF receptors with the Fc component of an IgG human antibody (Roche, Basel, Switzerland, sTNFR:Fc). After a three months placebo controlled phase she was treated with $50 \mathrm{mg}$ sTNFR:Fc every four weeks.

Results Clinical response was impressive with swollen joint counts decreasing from 32 to 5 and C-reactive protein CRP levels declining from 95 at baseline to 20 after the first injection. Low disease activity was sustained for the following years. Besides sTNFR:Fc her medication consisted of oral prednisone 5 $\mathrm{mg}$ a day and occasionally paracetamol $500 \mathrm{mg}$. In the spring of 1999 she first noticed nailfold infarcts on the fingers of both hands. These lesions disappeared after every injection of sTNFR: $F_{c}$ and reappeared three weeks thereafter when the clinical effects of sTNFR:Fc were decreasing. This effect on the digital vasculitis has been well documented during several cycles of sTNFR:Fc administration.

Conclusion The prompt disappearance of nailfold infarcts after sTNFR:Fc administration observed in our patient strongly suggests a therapeutic effect of sTNFR:Fc on active vasculitis. This observation raises the question whether blocking of TNF-alpha might also be effective in more severe forms of vasculitis and possibly other extra-articular manifestations of RA, some of which are life threatening and are currently treated with high doses of corticosteroids and immunosuppressive drugs.

\section{THU0195 INTRA-MACHINE AND INTRA-READER AGREEMENT OF RA DISEASE ACTIVITY AND DAMAGE USING MRI. COMPARISON WITH CLINICAL AND LABORATORY MEASURES}

P Bird, M Lassere. Rheumatology, St George Hospital, Sydney, Australia

10.1136/annrheumdis-2001.1097

Background Magnetic resonance imaging offers a direct means of evaluating inflammation disease activity and damage in rheumatoid arthritis joints. However, few studies have evaluated intra-machine MRI. ${ }^{1}$

Objectives Evaluate the agreement of intra-machine MRI using the OMERACT 5 Rheumatoid Arthritis MRI Score (Om5 RAMRIS) ${ }^{2}$ and compare MRI reliability to clinical and laboratory measures.

Methods 12 subjects with seropositive RA, median disease duration 6 years, were chosen to reflect spectrum of RA severity. MRI was performed at same time of day at baseline and $48 \mathrm{~h}$. All sources of variability were either controlled or measured. A cannula was inserted into the nondominant arm and serum was collected prior to MR for ESR/CRP. The cannula was left insitu for administration of gadolinium (Gd). MRI of wrist was performed with dominant hand splinted in semipronated position alongside the body. Axial and coronal images were obtained preGd using T1, proton density and T2 (FS) sequences. Gadolinium was administered at $0.1 \mathrm{mmol} / \mathrm{kg}$ over 90 secs. Post Gd fat suppressed sequences commenced 2 min post injection (axial then coronal). MRI images were read in random order by a single blinded observer using the Om5 RAMRIS. All images were reread in random order at $48 \mathrm{~h}$ by same observer. Additional clinical data at baseline and $48 \mathrm{~h}$ : HAQ, SF36, 28 swollen and tender joint count (same observer).

Results Intra-machine MRI (ie at baseline and $48 \mathrm{~h}$ ) intraclass correlation coefficients (ICCs) were lower for all measures of disease activity (synovitis global, synovial maximal thickness and bone oedema) than for lesions of disease damage (bone erosions and bone defects). ICCs of summated wrist activity score for intra-machine MRI were $0.53-0.70$, whereas intra-reader ICCs were 0.86 and 0.90 . However ICCs of summated wrist damage score for intra-machine MRI were $0.89-0.96$ and for intrareader were 0.93 and 0.97 . Mean (SD) scores at baseline and 48 hrs. ICCs for clinical and laboratory data were: DAS28 $=0.94$, $\mathrm{HAQ}=0.96, \mathrm{TJC}=0.89, \mathrm{ESR}=0.76, \mathrm{SJC}=0.72$ and $\mathrm{CRP}$ $=0.55$. Mean differences at baseline and $48 \mathrm{hrs}$ for all clinical, laboratory and MRI measures were not statistically significant. Furthermore, none of the differences consistently reflected either an increase or decrease in disease activity. Smallest detectable difference (SDD) for selected measures were as follows: wrist activity $\pm 35(50 \%$ of score range $(\mathrm{SR}))$, wrist damage $\pm 18(15 \%$ SR), DAS28 土. 98 (23\% SR), HAQ \pm .49 (21\% SR), SJC \pm 5.4 $(57 \% \mathrm{SR}), \mathrm{TJC} \pm 5.2(35 \% \mathrm{SR})$ and $\mathrm{CRP} \pm 10(71 \% \mathrm{SR})$.

Conclusion Intra-reader agreement using the OMERACT 5 Rheumatoid Arthritis MRI Score has excellent intra-reader agreement for the assessment of synovitis, bone oedema, bone erosions and defects. Intra-machine agreement at $48 \mathrm{~h}$ was also excellent for bone erosions and defects and the SDD was quite acceptable. Lower ICCs for measures of MRI RA activity probably reflect underlying true variability in disease activity even at $48 \mathrm{~h}$ (suggested by variability in CRP), as well as a component of measurement error, (implied by relative stability of DAS28). Thus to disentangle the discrimination (reliability vs responsiveness) of this MRI activity scoring system, it requires further evaluation in a RCT. 